\title{
Polyhedral linkages obtained as assemblies of planar link groups
}

\author{
Gökhan KİPER ${ }^{1}$, Eres SÖYLEMEZ ${ }^{2}$ \\ ${ }^{1}$ İzmir Institute of Technology, Turkey, e-mail: gokhankiper@iyte.edu.tr \\ ${ }^{2}$ Middle East Technical University, Turkey
}

\begin{abstract}
The study aims to devise means of obtaining polyhedral linkages for homothetic deployment of polyhedral shapes by embedding planar link groups in faces of the polyhedral shape of interest. The questions of which polyhedral shapes may be suitable for such a purpose and what are the compatibility conditions for spatially assembling planar link groups are addressed. Homohedral and tangential polyhedral shapes are found to be suitable for the task and some examples of linkages are worked out.
\end{abstract}

Key words: Polyhedral linkages, homothety, homohedra, tangential polyhedra

\section{Introduction}

Polyhedral linkages with invariant faces and variable angles were of interest in the first half of the $20^{\text {th }}$ century $[1,2,3]$. However in the last few decades researchers started to work on polyhedral linkages with variable faces, invariant dihedral and planar angles. Due to invariant angles these linkages will be called conformal polyhedral linkages.

An assembly with such a conformal transformation is first discovered by Buckminster Fuller [4]. Fuller named the motion as the Jitterbug motion [5]. Many Jitterbug-like linkages, were discovered later on. Verheyen [6] presented a list of regular Jitterbug-like linkages. Wohlhart discovered several Jitterbug-like linkages [7, 8, 9]. Röschel used planar equiform motions reflected on different planes to construct Jitterbug-like linkages $[10,11,12]$. In [11] Röschel applied his methods to tangential octahedra (octahedra with an inscribed sphere). Kiper and Söylemez generalized Röschel's findings and presented some other methods to obtain new Jitterbug-like linkages [13]. [13] specifically issues homothetic polyhedral linkages, for which the polyhedral volume inside the linkage remains scaled at all poses.

Other than the Jitterbug-like linkages, Gosselin and Gagnon-Lachance [14] and Kiper, Söylemez and Kişisel $[15,16]$ obtained conformal polyhedral linkages by implementing a single degree-of-freedom (dof) link group in all faces of a polyhedron while Wohlhart [17, 18, 19, 20, 21] and Kiper and Söylemez [22] used multi dof link groups on the faces. In all these linkages only revolute joints were used. In this study we aim to obtain homothetic polyhedral linkages with revolute joints only assembling appropriate link groups placed on the faces of polyhedra.

\section{Planar Link Groups}

Consider a polyhedral shape going through a homothety (non-rotating dilation). In practice, such a transformation of the polygonal faces can be realized by planar kinematic chains with $n$ prismatic joints along the edges actuated at rates proportional to the respective edge lengths (Fig. 1). 


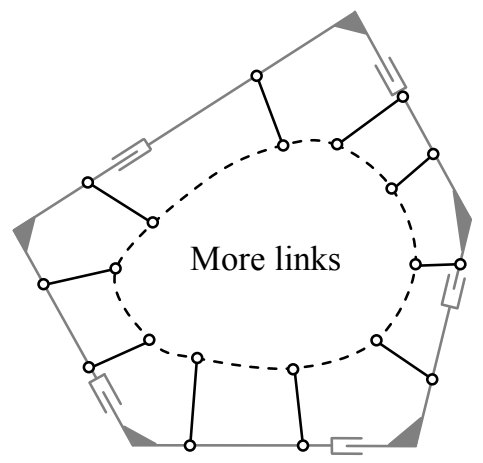

Fig. 1 Polygonal kinematic chain

When the revolute joints at the edges connect to the so-called dihedral angle preserving (dap) links, the planar chains are assembled to form a polyhedral linkage (See [13]). A necessary condition to be able to assemble such planar chains to obtain a polyhedral linkage is that the dap link connections of planar link groups in neighboring faces must be compatible. That is, adjacent planar link groups should be positioned at concurrent locations along the common edge at all times of motion. Such a condition necessitates special dimensioning both the polyhedral shape of interest and the links. Therefore the resulting linkages are overconstrained. Röschel [11, 12] and Kiper and Söylemez [13] have illustrated that this compatibility condition at the edges becomes possible when planar homothety centers of adjacent dilating face pairs are located such that the perpendiculars dropped from these centers to the edges meet each other. This condition is valid for the following two types of polyhedral geometry: tangential polyhedra and homohedra with edge-symmetric faces (See [13]).

Assume that we have a polyhedron for which one can pick a point on each face of a polyhedron such that the perpendiculars dropped from these points to the edges meet each other. These points shall be called planar homothety centers (PHCs). We need to assure that planar link groups (in the form of Fig. 1) keep the homothety centers invariant. To simplify things assume that in all planar linkages a link rotates about the corresponding homothety center, or keeps still with respect to this center. The links should be dimensioned and positioned such that the sides can uniformly dilate from the PHC. Especially using scissor-like link pairs and parallelograms are ideally suited for such a task. Three planar link chain examples are presented in Fig. 2. These chains can be classified according to how the distance of the PHCs to the sides is computed: variable + fixed distance (Ex. Fig. $2 \mathrm{a}:-|\mathrm{OA}| \cos \left(\alpha_{\mathrm{AB}}+\theta\right)$ as variable and $|\mathrm{AB}|$ as fixed distance), fixed + variable distance (Fig. 2b) and variable distance (Fig. 2c) from a PHC to the sides.

For the link group in Fig. 2a, the central link rotates about PHC O and the other links have translatory motion such that one side of each link remains on a polygon side. Example in Fig. 2a is for triangular dilation, yet in general, a dilation of an $n$-gon can be obtained when $n$ -1 constraints in the following form are satisfied:

$$
\begin{aligned}
\frac{|\mathrm{OE}|\left(\theta_{1}\right)}{|\mathrm{OE}|\left(\theta_{2}\right)}= & \frac{|\mathrm{OF}|\left(\theta_{1}\right)}{|\mathrm{OF}|\left(\theta_{2}\right)} \Rightarrow \frac{-|\mathrm{OA}| \cos \left(\alpha_{\mathrm{AB}}+\theta_{1}\right)+|\mathrm{AB}|}{-|\mathrm{OA}| \cos \left(\alpha_{\mathrm{AB}}+\theta_{2}\right)+|\mathrm{AB}|}=\frac{-|\mathrm{OC}| \cos \left(\alpha_{\mathrm{CD}}+\theta_{1}\right)+|\mathrm{CD}|}{-|\mathrm{OC}| \cos \left(\alpha_{\mathrm{CD}}+\theta_{2}\right)+|\mathrm{CD}|} \\
& \Rightarrow \quad \frac{|\mathrm{OA}|}{|\mathrm{AB}|}=\frac{|\mathrm{OC}|}{|\mathrm{CD}|}
\end{aligned}
$$


The construction in Fig. $2 b$ is rather a simple one. Parallelogram loops are attached to a polygonal link which is in similar to the polygonal face of interest (quadrilateral in Fig. 2b, any polygon in general). The parallelogram loops can be attached anywhere on the sides of the polygonal link. Condition for a homothety is found as:

$$
\begin{aligned}
\frac{|\mathrm{OE}|\left(\theta_{1}\right)}{|\mathrm{OE}|\left(\theta_{2}\right)}=\frac{|\mathrm{OF}|\left(\theta_{1}\right)}{|\mathrm{OF}|\left(\theta_{2}\right)} & \Rightarrow \frac{|\mathrm{OA}|+|\mathrm{AB}| \cos \theta_{1}}{|\mathrm{OA}|+|\mathrm{AB}| \cos \theta_{2}}=\frac{|\mathrm{OC}|+|\mathrm{CD}| \cos \theta_{1}}{|\mathrm{OC}|+|\mathrm{CD}| \cos \theta_{2}} \\
& \Rightarrow \frac{|\mathrm{OA}|}{|\mathrm{AB}|}=\frac{|\mathrm{OC}|}{|\mathrm{CD}|}
\end{aligned}
$$

The linkage in Fig. 2c has two identical polygonal links in the central region for which the sense of rotation is in opposite directions. This design can be considered to be derived from a Jitterbug-like linkage by addition of scissor pairs between the polygonal and dap links. It is possible to add more scissor pairs in series. The dilation ratio is

$$
\frac{|\mathrm{OE}|(\theta)}{|\mathrm{AB}|(0)}=\frac{(|\mathrm{OA}|+|\mathrm{AB}|+|\mathrm{BC}|) \cos \theta}{|\mathrm{OA}|+|\mathrm{AB}|+|\mathrm{BC}|}=\cos \theta
$$



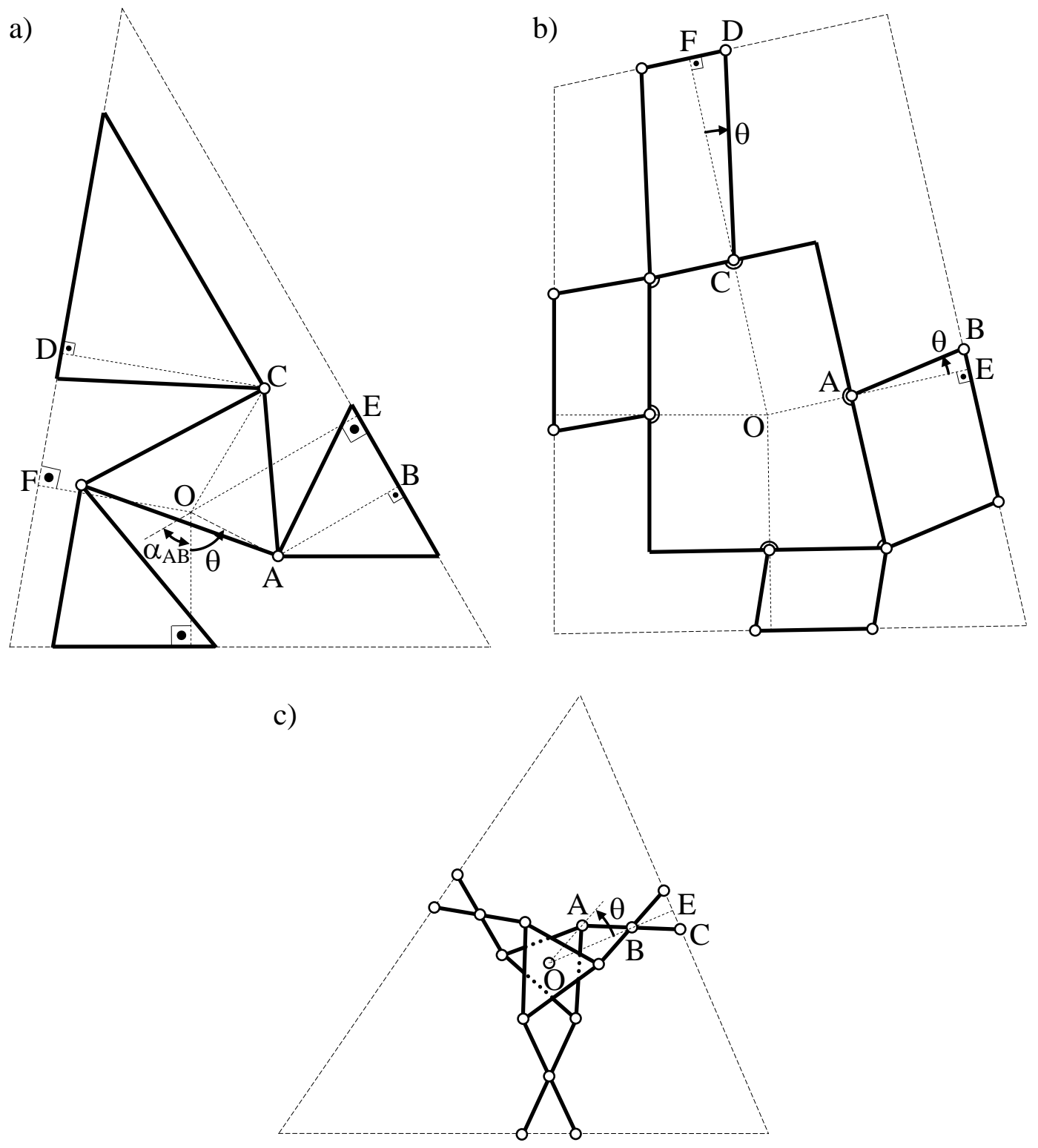

Fig. 2 a) variable + fixed length example b) fixed + variable length example, c) variable + variable length example

In the following two sections we use these planar link groups in assembling homohedral and tangential polyhedral linkages.

\section{Homohedral Linkages}

A homohedron is a polyhedron with congruent faces [22]. The 5 regular Platonic solids, the 13 semi-regular Archimedean solids, the 13 Catalan solids, the 8 deltahedra are well known homohedra. Not necessarily, but for most of homohedra adjacent faces are symmetric with respect to the common edge, so they are suitable for our purposes. Since adjacent faces are mirror symmetric, mirror symmetric link groups can be embedded in the faces. The planar link group in Fig. 2a is used for a regular octahedron as a homohedral linkage example (Fig. $3)$. 


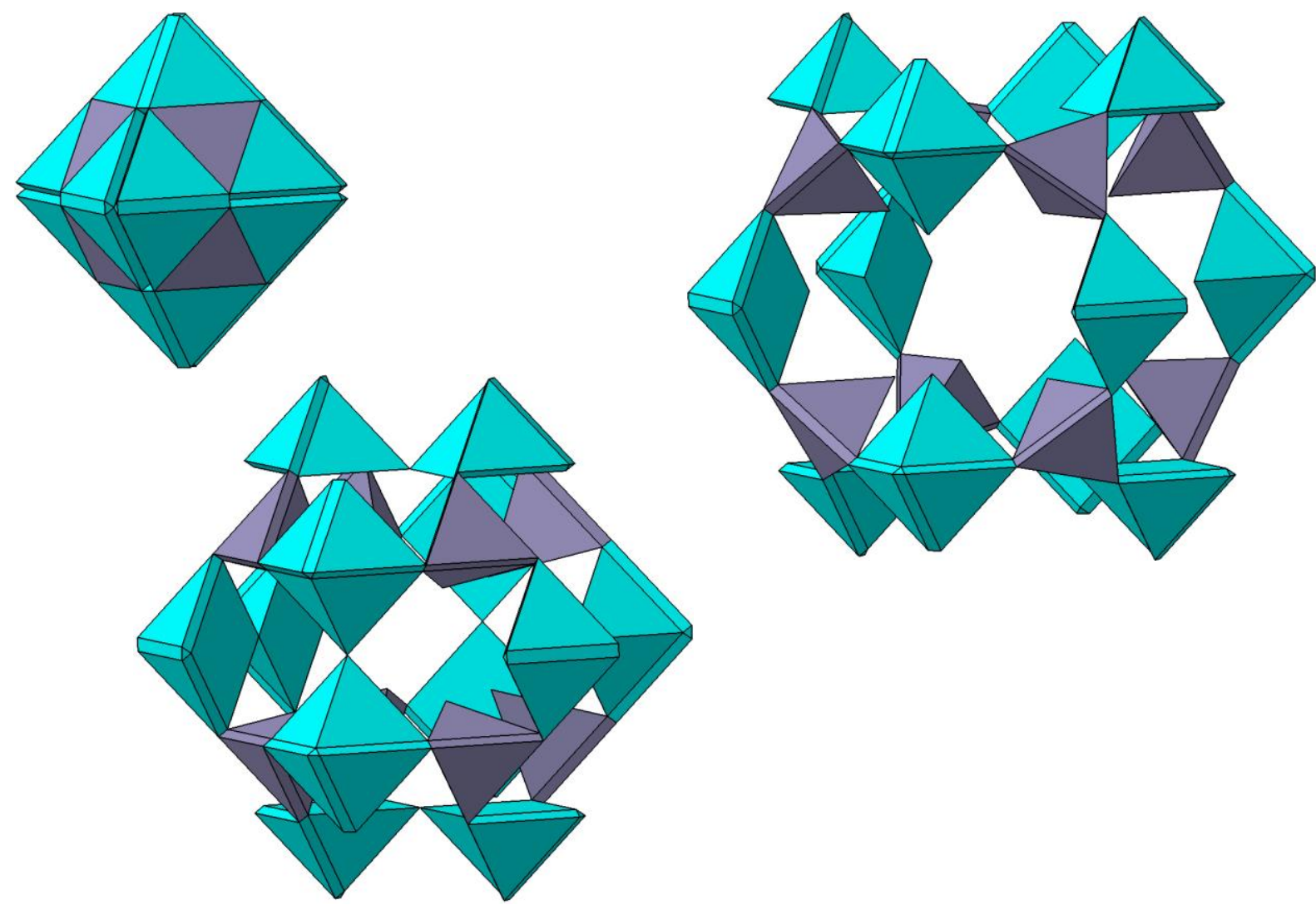

Fig. 3 Octahedral linkage in compact, deployed and further actuated forms

Note that such an octahedral linkage exactly performs the Jitterbug motion. All Jitterbuglike linkages can be modified in the same manner. There is no novelty in the kinematic structure in this example, however the dimensional design allows that the whole volumetric region inside the linkage is filled in the compact configuration. The links can be dimensioned in various ways (Fig. 4).
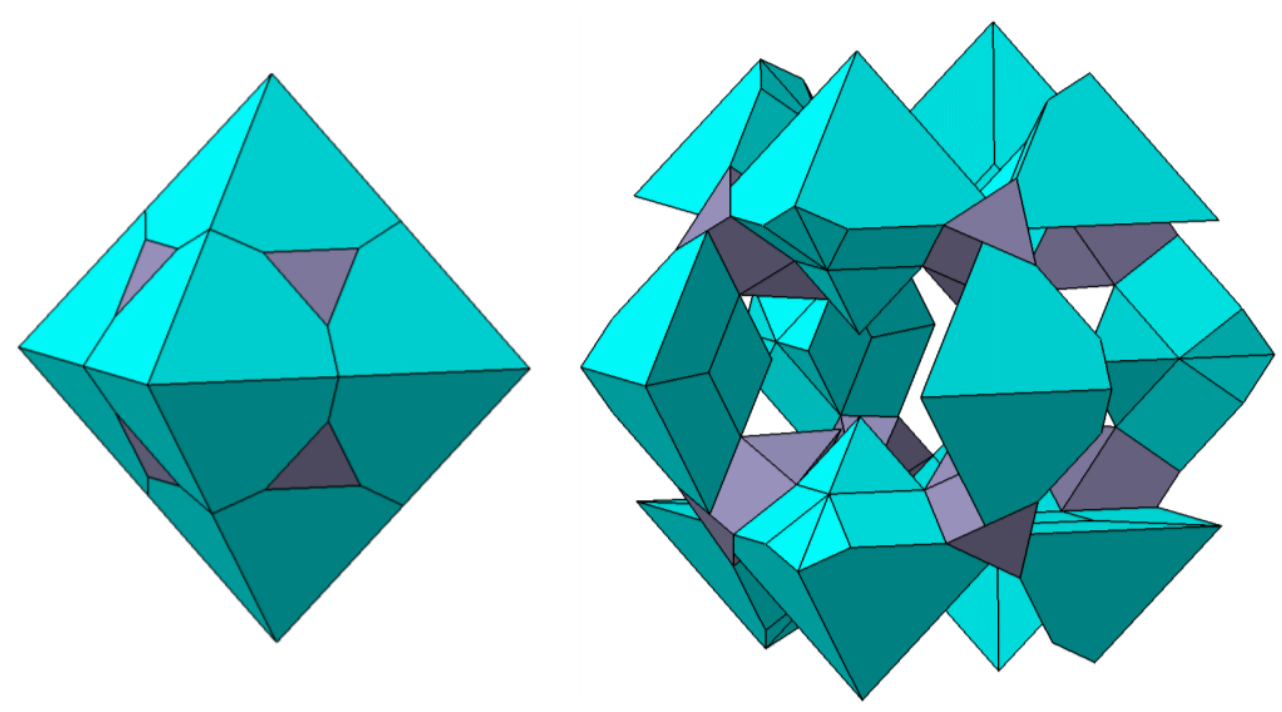
Fig. 4 Octahedral linkage with different dimensioning in compact and deployed forms

\section{Tangential Polyhedral Linkages}

Consider a polyhedron with an inscribed sphere. The perpendiculars dropped to the edges from the points of tangencies on the faces meet at the edges and also that points of tangencies on adjacent faces are equidistant to the common edge. To see that this is the case we shall use Fig. 5. In Fig. 5, $|\mathrm{OA}|=|\mathrm{OB}|$ which is equal to the radius of the insphere. The line of intersection of the adjacent faces that are necessarily tangent to the insphere meets the plane constructed by the lines OA and OB perpendicularly, hence the perpendiculars dropped from $\mathrm{E}$ to the intersection line meet at a point E. OAEB defines a kite, so $|\mathrm{AE}|=|\mathrm{EB}|$. See [13] for the details of the proof.

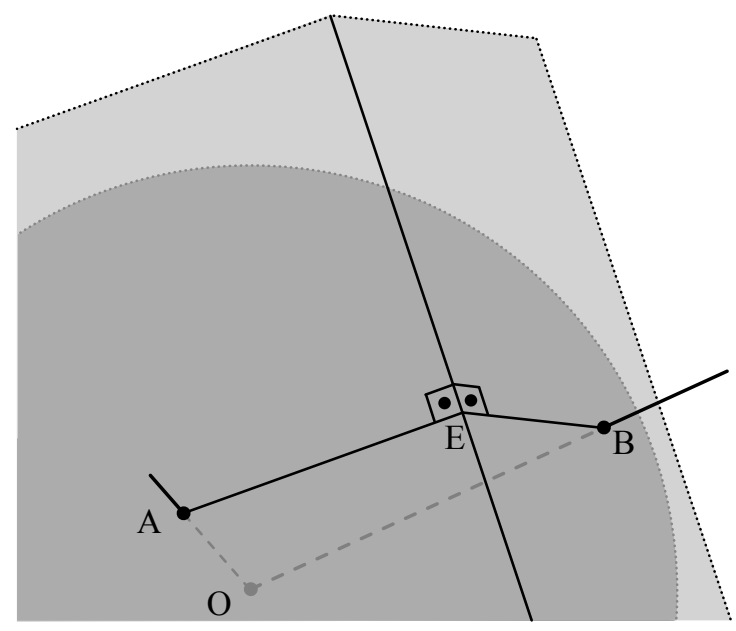

Fig. 5 Adjacent faces of a tangential polyhedron

If one can place planar link groups on the faces of such a tangential polyhedron such that the points of tangencies remain equidistant to the edge defined by the joints at the edges, planar dilation, hence polyhedral dilation is obtained.

A tetrahedral (all tetrahedra have inspheres) and a hexahedral tangential linkage using the planar chains in Fig. 2b, c are given in Figs. 6-7. For the tetrahedral linkage in Fig. 6, the instantaneous screw axes (ISAs) of the central links on the faces meet at the insphere center at all times, hence the ISAs remain still with respect to each other. This is proved in [13]. 

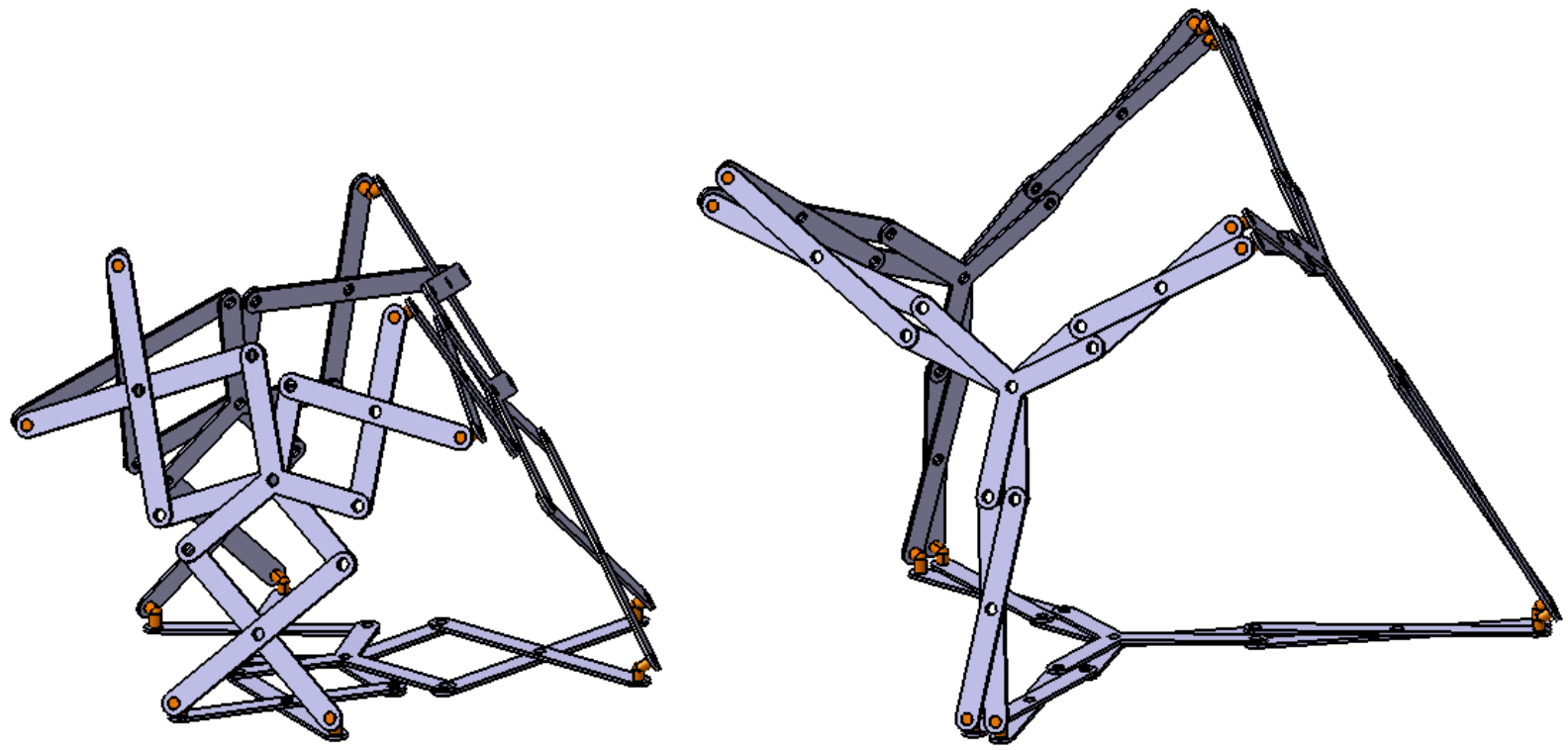

Fig. 6 Tetrahedral linkage in compact and deployed forms
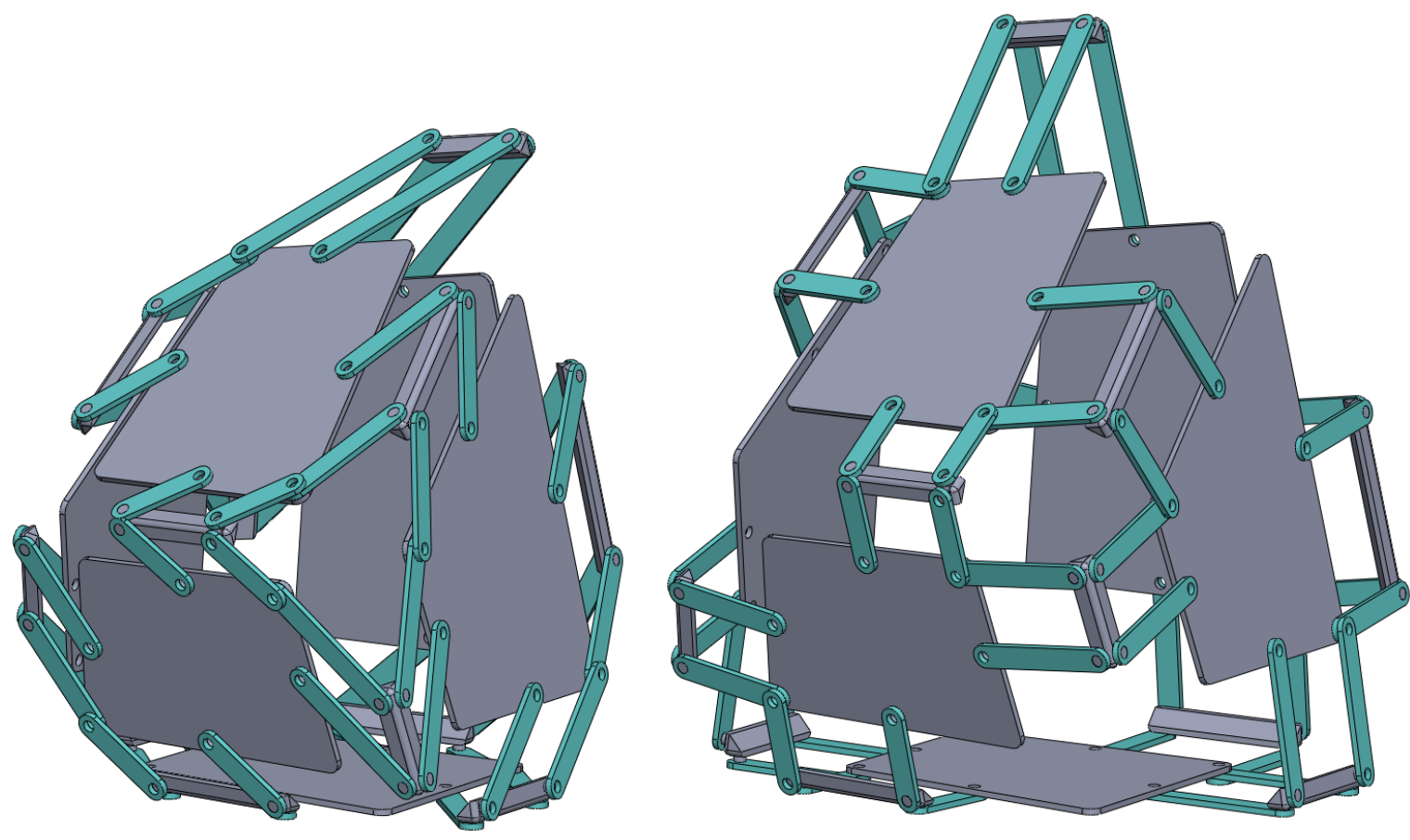

Fig. 7 Hexahedral linkage in compact and deployed forms

When the planar link groups used for the hexahedral linkage are used for more symmetric figures, ex. the cube, the surface of the polyhedral shape can be fully closed in the physically obtainable compact form (Fig. 8). 

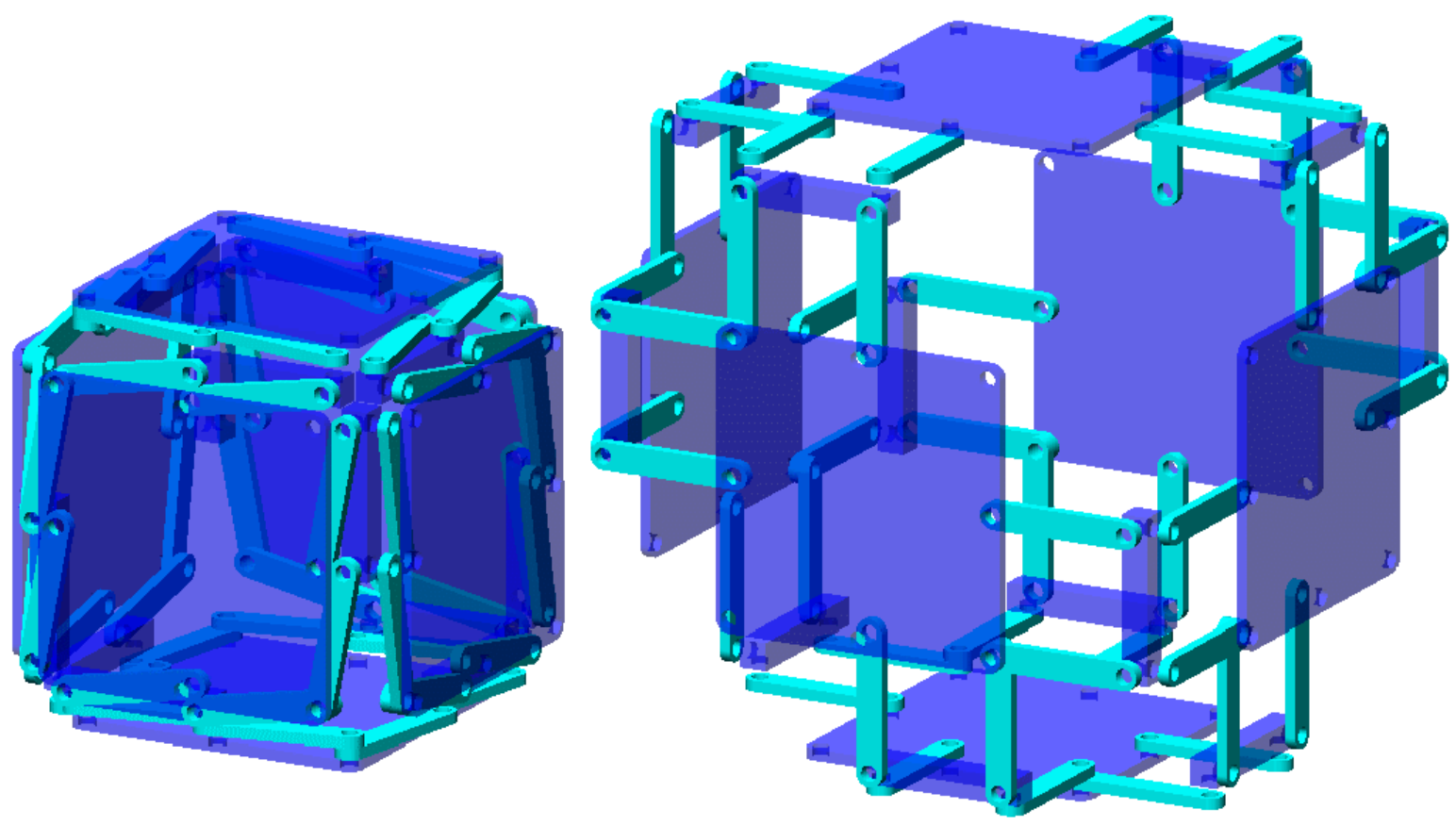

Fig. 8 Cubic linkage in compact and deployed forms

\section{Conclusions}

Conformal polyhedral linkages with multi link planar chains on the faces are issued in this study. Planar link groups are designed such that the elements on the polygonal boundaries will comply when the spatial linkage is assembled. Homohedra and tangential polyhedra are used to obtain single dof linkages. Many other types of conformal polyhedral linkages can be found following similar steps in this paper. Discovery of other types of feasible polyhedral geometries remains as an open problem.

\section{References}

1. Bricard R. Mémoire sur la théorie de l'octaèdre articulé. J Math Pures Appl, 1897, 3: 113-150

2. Bennett G T. Deformable octahedra. Proc London Math Soc, $2^{\text {nd }}$ Series, 1911, 10: 309-343

3. Goldberg M. Polyhedral linkages. Nat Math Mag, 1942, 16: 323-332

4. Fuller R B, Krausse J, Lichtenstein C. Your Private Sky: R. Buckminster Fuller, the Art of design Science. Lars Müller, 1999

5. Fuller R B. (1975). Synergetics: Explorations in the Geometry of Thinking. Macmillan, 1975, Section 460.

6. Verheyen H F. The complete set of Jitterbug transformers and the analysis of their motion. Int J Comput Math Appl, 1989, 17: $203-250$

7. Wohlhart K. Heureka octahedron and Brussels folding cube as special cases of the turning tower. In: Proceedings of the $6^{\text {th }}$ IFToMM International Symposium on Linkages and Computer Aided Design Methods, Bucharest, 1993, 325-332

8. Wohlhart K. The screwtower, an overconstrained multi-loop space mechanism. In: Proceedings of the International Conference on Spatial Mechanisms and High-Class Mechanisms, 1, Almaty, 1994, 38-45

9. Wohlhart K. New overconstrained spheroidal linkages. In: Proceedings of the $9^{\text {th }}$ IFToMM Congress, Milano, 1995, 149155

10. Röschel O. Zwangläufig bewegliche Polyedermodelle I. Math Pann, 1995, 6: 267-284

11. Röschel O. Zwangläufig bewegliche Polyedermodelle II. Stud Sci Math Hung, 1996, 32: 383-393

12. Röschel O. Zwangläufig bewegliche Polyedermodelle III. Math Pann, 2001, 12: 55-68 
13. Kiper G, Söylemez E. Homothetic Jitterbug-like linkages. Mech Mach Theory, 2012, 51: 145-158

14. Gosselin C M, Gagnon-Lachance D. Expandable polyhedral mechanisms based on polygonal one-degree-of-freedom faces. Proc Inst of Mech Eng Part C Mech Eng Sci, 2006, 220: 1011-1018

15. Kiper G, Söylemez E, Kişisel A U Ö. A family of deployable polygons and polyhedra. Mech Mach Theory, 2008, 43: $627-640$

16. Kiper G, Söylemez E. Irregular polygonal and polyhedral linkages comprising scissor and angulated elements. In: Proceedings of the $1^{\text {st }}$ IFToMM Asian Conference on Mechanism and Machine Science (CD), Taipei, 2010

17. Wohlhart K. Regular polyhedral linkages. In: Proceedings of the $2^{\text {nd }}$ Workshop on Computational Kinematics, Seoul, 2001, 239-248

18. Wohlhart K. New regular polyhedral linkages. In: Proceedings of the SYROM 2001, Bucharest, 2001, 365-370

19. Wohlhart K. Irregular polyhedral linkages, In Proceedings of the $11^{\text {th }}$ World Congress in Mechanism and Machine Sciences, Tianjin, 2004, 1083-1087

20. Wohlhart K. Double pyramidal linkages. Proccegings of the $11^{\text {th }}$ International Symposium on Theory of Machines and Mechanisms, 1, Bucharest, pp. 293-300 (2005)

21. Wohlhart K. Double-ring polyhedral linkages. In: Proceedings of the Conference on Interdisciplinary Applications of Kinematics, Lima, pp. 1-17 (2008)

22. Kiper G, Söylemez E. Obtaining new linkages from Jitterbug-like polyhedral linkages. In: Proceedings of the AzC IFToMM 2010 International Symposium of Mechanism and Machine Science, İzmir, 2010, 137-143

22. Olshevsky G. Glossary for Hyperspace (2006) available via http://web.archive.org/web/ 20070207021813/http://members.aol.com/Polycell/glossary.html 